\title{
TIME DOMAIN SIMULATIONS OF THE CLIC PETS (POWER EXTRACTION AND TRANSFER STRUCTURE) WITH GdfidL
}

\author{
I. Syratchev, CERN, Geneva, Switzerland \\ W. Bruns, TU-Berlin, Germany
}

\begin{abstract}
The Compact Linear Collider (CLIC) PETS [1] is required to produce about $0.5 \mathrm{GW}$ RF power per metre in the $30 \mathrm{GHz}$ CLIC decelerator when driven by the high current beam $(\sim 270 \mathrm{~A})$. To avoid beam break-up in the decelerator it is necessary to provide strong damping of the transverse deflecting modes. A PETS geometry with a level of damping consistent with stable drive beam operation has been designed, using the frequency domain code HFSS. A verification of the overall performance of this structure has been made recently using the code GdfidL, which permits a very fine mesh analysis of a fulllength structure in the time domain. This paper gives the results of this analysis.
\end{abstract}

\section{INTRODUCTION}

Time domain simulations of RF fields in travelling wave structures give more precise information about the wakefield behaviour than the frequency domain approach. A time domain simulation of the CLIC PETS has been made using GdfidL [2]. This particular code was chosen because it has the ability to calculate dielectric losses and can calculate very long structures (about 100 cells) in a reasonable time. The ability to compute long structures is necessary in order to differentiate the RF losses from the natural decay associated with high group velocity structures. The results of these simulations are given in this paper.

\section{EM CODE GDFIDL}

GdfidL is a finite difference based code, which restricts computation to the field-carrying parts of the computational volume. In the case of the PETS, this means that GdfidL has to compute the fields in only $30 \%$ of the total number of grid cells. Even though GdfidL computes efficiently, for a structure as large as the PETS, current single-processor systems are far too slow to compute the electromagnetic fields in a reasonable time and the use of multiprocessor systems becomes mandatory. The most powerful multiprocessor systems are distributed memory systems, like the Cray T3E. GdfidL running on such systems partitions the total computational domain in sub-domains. For every timestep, all processors simultaneously compute the electromagnetic field in their local sub-domain and indicate to each other the tangential fields on their local outer boundaries. The measured performance of the parallel version of GdfidL on 100 processors of a T3E is about 50 times the performance of a $1 \mathrm{GHz}$ AMD-Athlon.

\section{SIX-WAVEGUIDE PETS}

A 6-waveguide version of PETS with large $(30 \mathrm{~mm})$ beam aperture has been designed (Fig. 1) to satisfy the low-decelerating-impedance requirement for the CLIC two-beam scheme. To avoid beam break-up in the decelerator, strong damping of the transverse modes is needed. The most dangerous mode is the first dipole mode, which has practically the same frequency as the decelerating mode because in this particular PETS geometry most of the energy is concentrated around the waveguides. As a consequence, the usual damping method for a classical accelerating structure, based on a frequency-selective interception of the longitudinal wall current, could not be used. The damping scheme adopted is shown in Fig. 1. Corrugated longitudinal slits intercept the transverse image current of the TE-type hybrid mode [1]. Since the density of the dipole mode current in the slot area is rather weak, the coupling of the dipole mode to the damper was improved by making the slot and $\mathrm{SiC}$ damper geometry resonant at $30 \mathrm{GHz}$. The structure was optimized using the frequency domain code HFSS. It was shown that the $Q$-factor of unwanted transverse mode around $30 \mathrm{GHz}$ was reduced to below 50 [3].

To verify the presence of this strong damping, simulations of a 100-cell structure plus RF output couplers were done with GdfidL.

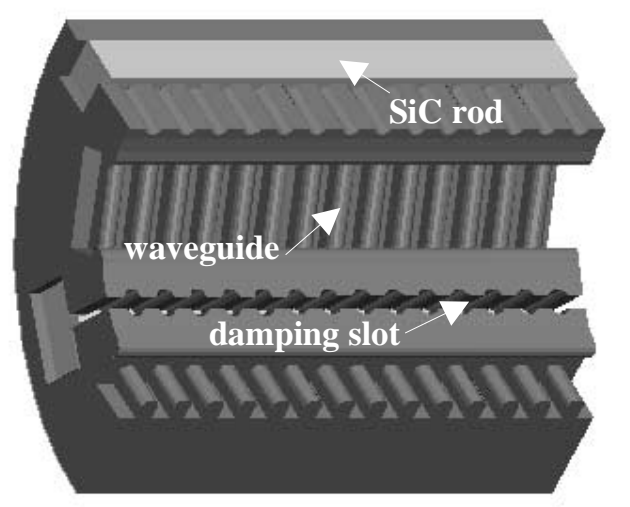

Figure 1. The quarter geometry of the 6-waveguide PETS.

The damping observed with GdfidL was over 10 times less than predicted with HFSS. The error was due to a wrong interpretation in HFSS of the resonant character of the transverse mode coupling to the damper.

The GdfidL simulation also showed the presence of low-frequency trapped modes. These modes are simply the cut-off modes of the PETS beam aperture and are not 
damped. Modes associated with the output coupler were also found.

Since the level of damping in the 6-waveguide structure was found to be insufficient ( 1000), an alternative design based on a structure with circular symmetry was undertaken. This design is discussed in the next chapter.

\section{CIRCULARLY SYMMETRIC PETS}

Earlier attempts [4] to design a circularly symmetric PETS were abandoned because no satisfactory method was found to damp the high group velocity transverse modes. This was particularly problematic because their frequencies are very close to the frequency of the main mode. However, as a result of a fairly even angular distribution of the RF current on the surface of the circular structure, it is clear that the damping does not need to be resonant.

A new concept of distributed damping of the transverse modes in a circularly symmetric structure has been developed. It is best explained in terms of array antennas. The damping is provided by thin longitudinal slots running all along the length of the structure. Every period of the slot-loaded structure acts like a single source in an array antenna, radiating through the slots to the outside. The radial component of the radiation (damping) is a function of the phase advances between two cells. The smaller the phase advance, the stronger the damping. Broadband RF loads terminate the slots.

\subsection{HFSS results}

As an example, a structure with a smooth, sinusoidal corrugation of the $20 \mathrm{~mm}$ beam aperture, C-PETS (circular), was studied. The damping was provided by 12 equally-spaced radial slots of $0.5 \mathrm{~mm}$, as shown in Fig. 2 . The 12 loads each consisted of a long $\mathrm{SiC}$ rod with a triangular cross-section which was matched to the slot.

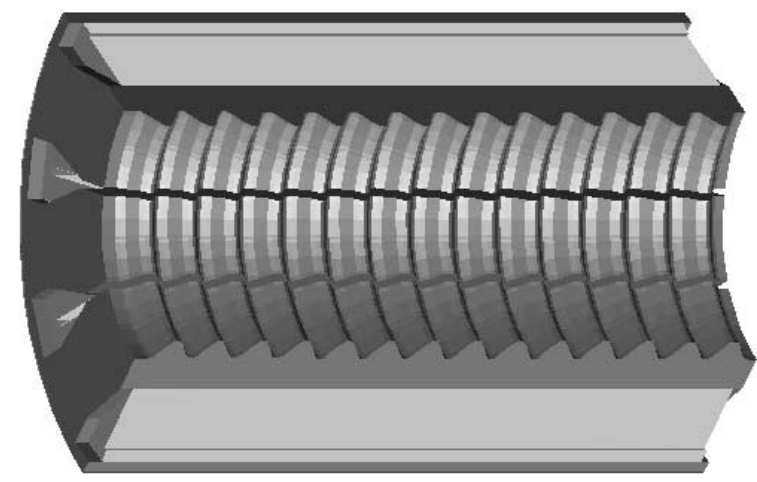

Figure 2. A quarter geometry of the C-PETS with 12 damping slots and $\mathrm{SiC}$ loads.

The slot depth and load geometry were chosen to minimize the external $Q$-factor of the first dipole mode around $30 \mathrm{GHz}$. The parameters for the optimised geometry are shown in Table 1 and Fig. 3. A very strong damping of the dipole mode is seen, that increases as the phase advance is decreased, as expected.

Table 1. The first dipole mode parameters with and without distributed damping.

\begin{tabular}{|c|c|c|c|c|}
\hline & $\varphi^{\mathrm{O}}$ & $\begin{array}{c}F \\
(\mathrm{GHz})\end{array}$ & $Q$-factor & $\begin{array}{c}\beta_{\text {GROUP }} \\
(\% \mathrm{c})\end{array}$ \\
\hline Undamped & 121 & 30.312 & 12315 & 83.4 \\
\hline Damped & 121 & 30.133 & $\sim 30$ & 66.3 \\
\hline
\end{tabular}

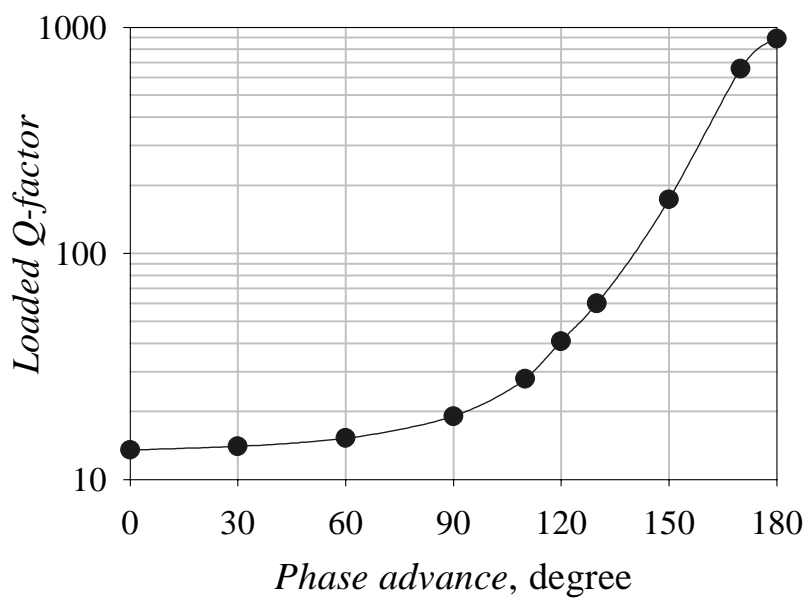

Figure 3. Damping of the dipole mode in C-PETS with 12 damping slots for the different phase advances per cell.

To make a qualitative study of the damping in the CPETS, a constant beam current excitation was introduced in HFSS.

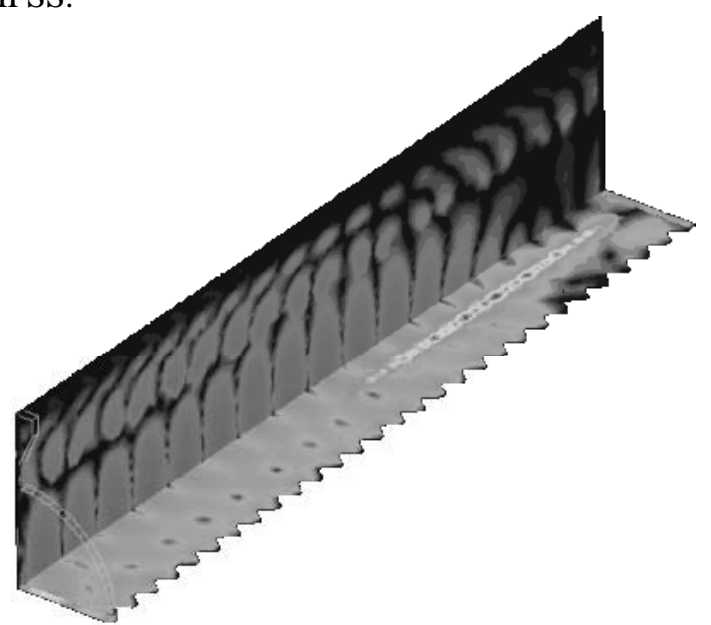

Figure 4. Electric field pattern in C-PETS excited by the beam.

The radial propagation of the RF field towards the load is clearly seen in Fig. 4 and indicates damping. Note that the same kind of simulations performed for the 6waveguide PETS did not show much radial RF power leak (damping). 


\subsection{GdfidL results}

The same 100-cell C-PETS geometry (see Fig. 2) was used to make simulations with GdfidL. A $1.5 \mathrm{~cm}$ long beam pipe was added to both ends. The computation of the wake-potentials up to distances of $4 \mathrm{~m}$ was made for $6 \times 10^{7}$ grid points. The computation time was 8 hours on 100 CPUs of a Cray T3E, or 75 hours on eight AMDAthlon-1000.

The short-range transverse wakes for the undamped and damped cases are shown in Fig. 5. The fast roll-off for the undamped case is caused by very fast evacuation of the RF power from the structure due to the high group velocity. This illustrates the importance of simulating long structures, especially with strong damping. For the damped case, the even further decay of the wake is a result of the strong damping. The loaded $Q$-factor in this case was calculated to be 35 . This value is very close to the one calculated with HFSS.

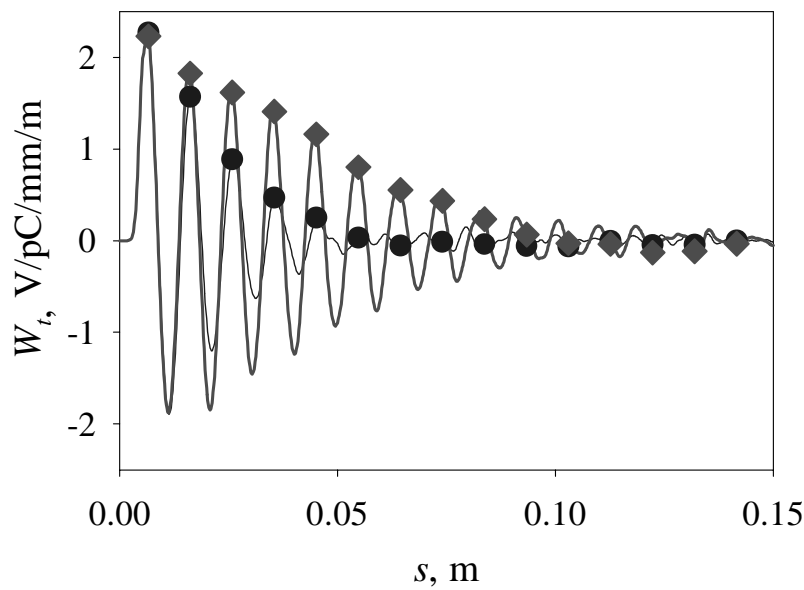

Figure 5. The short-range wakes in the C-PETS, diamonds: undamped case, circles: with damping.

The long-range transverse wakes and transverse impedances are shown in Figs. 6 and 7. The second big spike in the wake distribution for the undamped case (Fig. 6) is a result of partial (about $30 \%$ ) reflection from the ends of the structure. With damping the reflection is not seen due to the strong attenuation of the reflected wave.

From a comparison of the transverse impedances (Fig. 7) it is seen that low-frequency trapped modes are also significantly suppressed with this distributed damping technique.

\section{CONCLUSION}

The time-domain simulations of the full size 6-waveguide PETS with GdfidL showed that the damping of the transverse modes was strongly over-estimated with HFSS. A circularly symmetric version of the CLIC PETS structure is in consequence being reconsidered. With the novel concept of distributed damping, the strong suppression of the transverse modes in a broad frequency range has been demonstrated both by HFSS and GdfidL. The design of the C-PETS prototype together with RF couplers is now underway.

GdfidL was found to be well adapted for the simulation of large, complicated RF structures, like PETS, in a reasonable computing time.

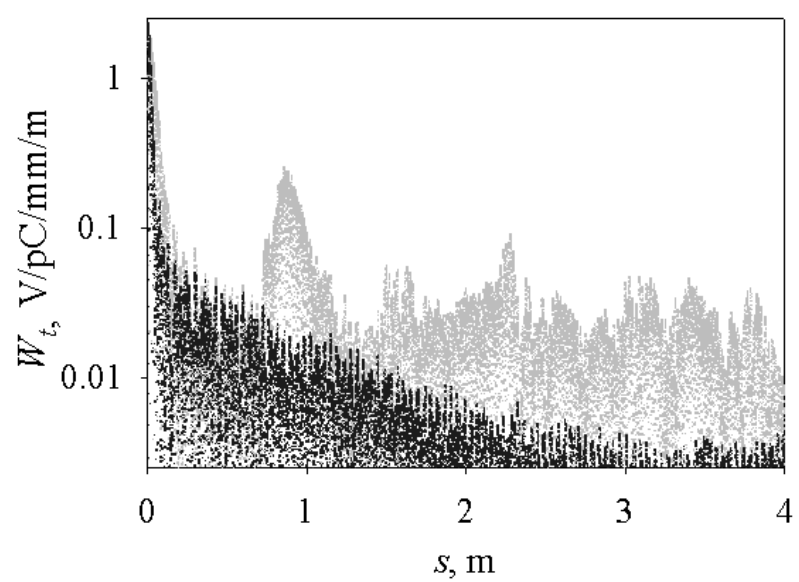

Figure 6. The long-range wakes in the C-PETS, grey: undamped case, dark: with damping.

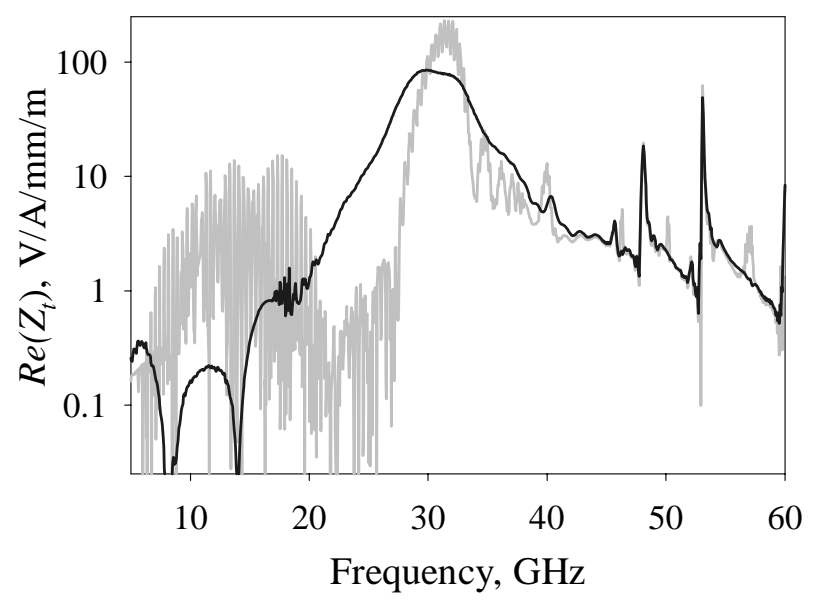

Figure 7. The transverse impedance in the C-PETS, grey: undamped case, dark: with damping

\section{REFERENCES}

[1] G. Guignard, et al., "A $3 \mathrm{TeV} \mathrm{e}^{+} \mathrm{e}^{-}$Linear Collider Based on CLIC Technology", CERN 2000-008, Geneva 2000.

[2] W. Bruns, "The GdfidL Electromagnetic Field Simulator", http://www.wbfb.de/.

[3] M. Luong, I. Syratchev, "Simulations of the Damping of the Power Extraction and Transfer Structure (PETS)", CLIC-Note-442, 2000.

[4] H. H. Braun, et al., "The CLIC RF Power Source", CERN 99-06, Geneva, 1999. 